\title{
Pretibial mucinosis in an euthyroid patient ${ }^{*}$
}

\author{
Laura de Mattos Milman ${ }^{1}$ \\ Giana Paula Müller ${ }^{1}$ \\ Paulo Ricardo Martins Souza ${ }^{1,2}$
}

\author{
Aline Barcellos Grill ${ }^{1}$ \\ Damiê De Villa ${ }^{1}$
}

DOI: http:/ / dx.doi.org/10.1590/abd1806-4841.20164043

\begin{abstract}
A case of exuberant pretibial mucinosis in a patient with normal thyroid function is reported. A review of literature on possible etiologies other than thyroid disease for the accumulation of mucin in the pretibial area is presented. In the patient described, it is possible that vascular insufficiency is involved. However, this is not the only factor responsible for the accumulation of mucin, since there are still unidentified causes and many patients with vascular diseases do not develop similar injuries.
\end{abstract}

Keywords: Myxedema; Mucinoses; Venous insufficiency

\section{INTRODUCTION}

Pretibial mucinosis is a build-up of dermal mucin in the front portion of legs. Although the terms myxedema and pretibial mucinosis have been used as synonyms, pretibial myxedema must be reserved for patients with thyroid disease. ${ }^{1}$ Other etiologies have been suggested for the accumulation of pretibial mucin, including cases of uncertain causes.

We report a case of pretibial mucinosis with exuberant clinical manifestations and discuss its possible causes in patients without thyroid dysfunction.

\section{CASE REPORT}

Female patient, 70 years old, phototype III, retired, sought medical attention due to 10-year-old lesions that progressively increased in number and size. She was a smoker, with a history of bilateral saphenectomy at 24 years of age and ischemic stroke at 62. A dermatological examination revealed erythematous papules of translucent surface, some of them confluent forming well-delimited plaques affecting mainly the pretibial region (Figure 1).
Laboratory investigation did not show alterations, including TSH, free T4, anti-peroxidase, anti-thyroglobulin and anti-TSH receptor antibodies. Echo-doppler of lower limbs showed venous and lymphatic insufficiency.

Histopathological examination demonstrated, with hematoxylin-eosin staining, loss of collagen-fiber deposition, discrete perivascular infiltrate, prominent fibroblasts and presence of hemosiderin; with Alcian Blue staining, mucin deposition across the entire dermal thickness (Figures 2 and 3).

\section{DISCUSSION}

Cutaneous mucinosis is a heterogeneous group of diseases caused by an abnormal accumulation of dermal mucin, an amorphous substance composed mainly of hyaluronic acid and glycosaminoglycans, uncommon in the pretibial region. ${ }^{1,2}$

Even though the terms pretibial myxedema and pretibial mucinosis have been frequently used as synonyms, pretibial myxedema is a kind of mucinosis 


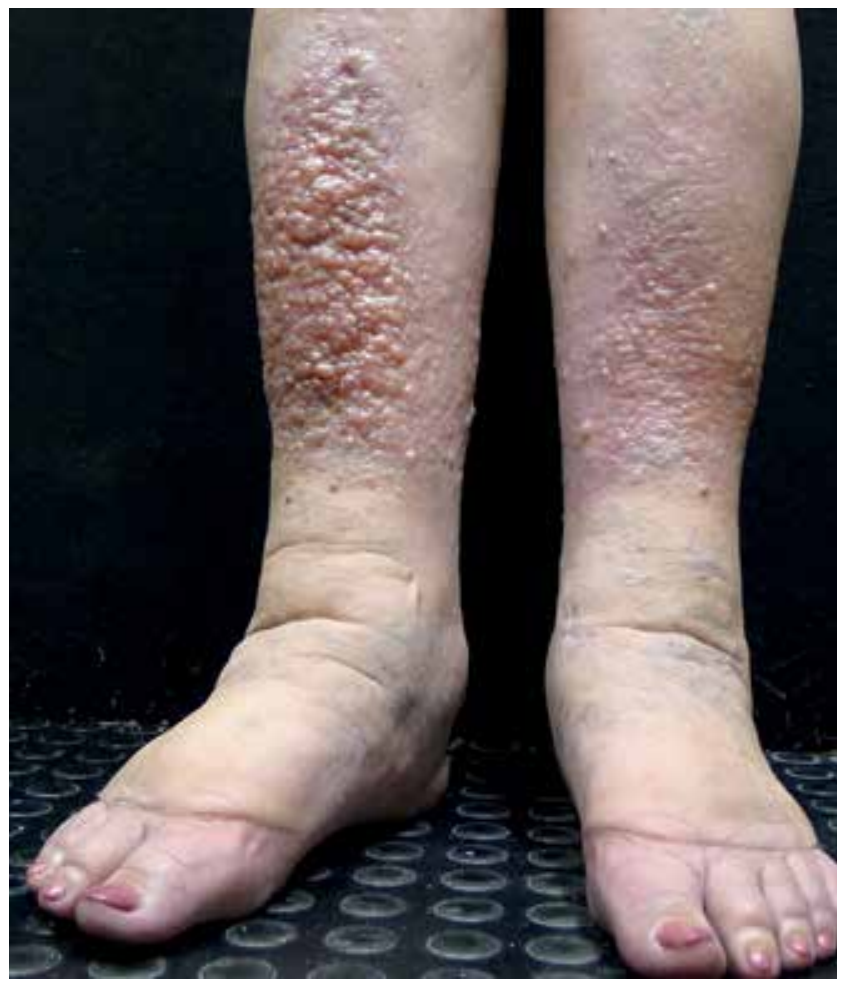

FIGURE 1: Translucent erythematous papules forming plaques in the pretibial region

associated with autoimmune thyroid disease. ${ }^{1}$

Pretibial myxedema is a rare cutaneous manifestation, occurring in up to $4 \%$ of patients with Graves disease, and less frequently in patients with Hashimoto's thyroiditis. ${ }^{1,3}$ Although usually limited to the pretibial area, it has also been described in other regions, with terms like thyroid dermopathy or localized myxedema being considered the most appropriate ones. ${ }^{1,3}$

In initial case reports of pretibial mucinosis in patients with normal thyroid function it was still questioned if they would develop thyroid disease in the future. ${ }^{4,5}$ Later other conditions that could lead to pretibial mucin deposition, among those trauma, venous insufficiency, lymphedema and obesity were described. ${ }^{1}$

In $2002 \mathrm{Kim}$ et al reported a case of pretibial mucinosis in a patient without evidence of autoimmune thyroid disease. This patient presented the symptom after a car crash trauma with subsequent venous insufficiency, lymphedema and recurring cellulitis on the lower limbs. ${ }^{6}$

In 2006 Tokuda et al reported 3 cases of pretibial mucinosis in patients with morbid obesity and chronic edema of lower limbs. ${ }^{7}$ In 2009 Rongioletti et al described 5 patients with similar symptoms to the ones described by Tokuda. ${ }^{8}$

In 2010 Pugashetti, $\mathrm{R}$ et al described two cases

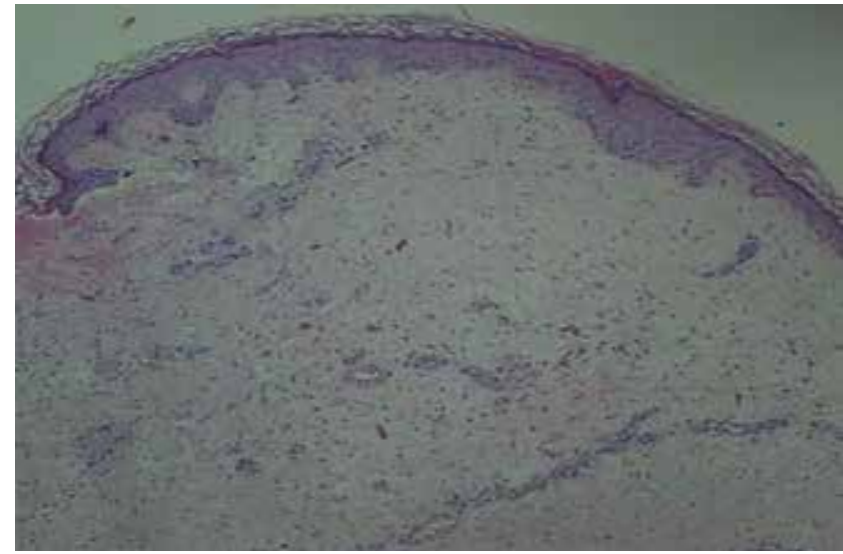

FIGURE 2: Loss of collagen fibers deposition, discrete perivascular infiltrate, prominent fibroblasts and presence of hemosiderin hematoxylin-eosin staining (50X augmentation)

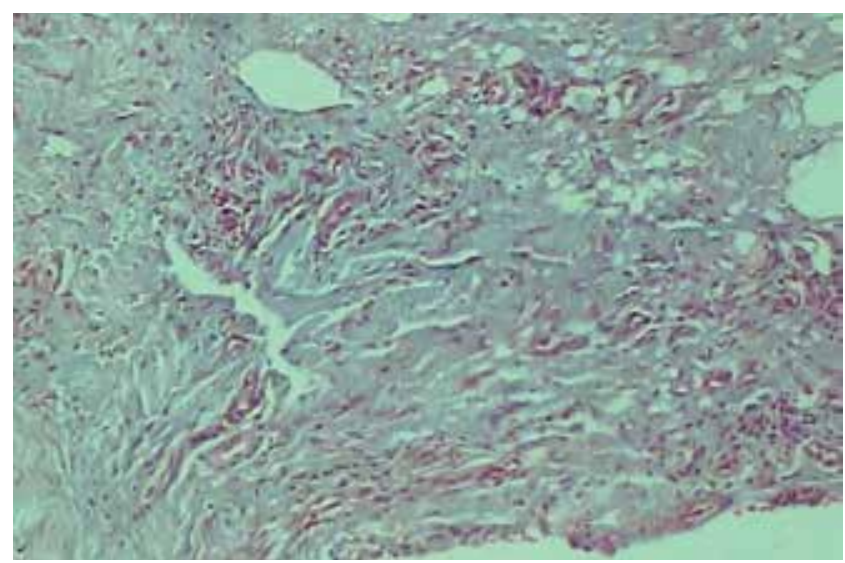

FigURE 3: Mucin accumulation across the entire thickness of dermis, Alcian Blue staining (100X augmentation)

of dermal mucinosis in the lower extremities as a sign of venous insufficiency.

The mechanism of dermal mucin accumulation in a stasis context is still uncertain, but hypoxia may be a determinant. $^{2}$ Venous insufficiency leads to decreasing levels of oxygen and extravasation of plasma proteins, such as albumin and coagulation factors. Formation of microthrombi worsens ischemia and triggers angiogenesis, while albumin and other plasma proteins increase production of mucopolysaccharides. Stasis in protein-rich lymph fluid in chronic lymphedema may stimulate mucin production in a similar way. ${ }^{1}$

In 2011, Mohsin et al reported a case of pretibial mucinosis in a patient without autoimmune thyroid disease, lymphedema, venous insufficiency, trauma or myxedematous lichen, suggesting that there may be other causes, not yet identified, responsible for pretibial mucin accumulation. ${ }^{1}$

In 1993 Somack et al reviewed biopsies of patients with pretibial mucinosis to try to determine histopathological characteristics capable of 
differentiating cases with and without thyroid disease. ${ }^{9}$

The most frequent histopathological features found in those with Graves disease were normal aspect of collagen in superficial papillary dermis and mucin deposition in the reticular dermis. In cases without thyroid disease, characteristic findings were mucin deposition in the superficial papillary dermis, angioplasty and presence of hemosiderin. Biopsies of patients with stasis were also evaluated; most of them presented accumulation of mucin in the papillary dermis and none in the reticular dermis.

That study suggested that pretibial mucinosis could occur in patients without thyroid disease. In these patients, histopathology showed both deposition of mucin and typical findings of stasis. They concluded that pretibial mucinosis could result from stasis or Graves disease and that the histopathological findings would allow an accurate differentiation of the two conditions. ${ }^{9}$ However, the differential diagnosis of pretibial mucinosis must not be restricted to these two pathologies in view of the existence of other etiologies already mentioned and cases of still uncertain etiology.
In the patient reported, venous and lymphatic insufficiency are probable contributors but are possibly not the only responsible for mucin accumulation, for not all causes of pretibial mucinosis have been properly described up to the present day and most patients with vascular insufficiency do not develop such exuberant lesions. More studies are necessary on the subject so that its causes can be more accurately identified.

As for the treatment, many options have been used with varied results. Topical corticoids applied with occlusion and compression therapy are local treatments which have been effective in reducing the lesions. Other options that present greater risk, such as intralesional and oral corticosteroids, as well as plasmapheresis, intravenous immunoglobulin, octreotide and pentoxifylline have shown unsatisfactory responses. Grafts and surgical excision have high rates of recurrence. More recently there has been a report of a carbon dioxide laser showing good results. ${ }^{3,10} \square$

\section{REFERENCES}

1. Mir M, Jogi R, Rosen T. Pretibial Mucinosis in a Patient Without Graves Disease Cutis. 2011;88:300-2.

2. Pugashetti R, Zedek DC, Seiverling EV, Rajendran P, Berger T. Dermal mucinosis as a sign of venous insufficiency. J Cutan Pathol. 2010;37:292-6.

3. Fatourechi V. Pretibial Myxedema - Pathophysiology and Treatment Option. Am J Clin Dermatol. 2005;6:295-309.

Chen JJ, Ladenson PW. Euthyroid pretibial myxedema. Am J Med. 1987;82:318-20. Srebrnik A, Ophir J, Brenner S. Euthyroid pretibial myxedema. Int J Dermatol. 1992;31:431-2

6. Kim KJ, Kim HH, Chang SE, Choi JH, Sung KJ, Moon KC, et al. A case of pretibial mucinosis without thyroid disease. J Dermatol. 2002;29:383-5.

7. Tokuda Y, Kawachi S, Murata H, Saida T. Chronic obesity lymphoedematous mucinosis: three cases of pretibial mucinosis in obese patients with pitting oedema. Br J Dermatol. 2006;154:157-61.

8. Rongioletti F, Donati P, Amantea A, Ferrara G, Montinari M, Santoro F, et al. Obesity - associated lymphoedematous mucinosis. J Cutan Pathol. 2009;36:1089-94

9. Somach SC, Helm TN, Lawlor KB, Bergfeld WF, Bass J. Pretibial mucin: histologic patterns and clinical correlation. Arch Dermatol. 1993;129:1152-6.

10. Castiñeiras I, del Pozo J, Robles 0, Martínez-González C, Fernández-Torres R, Fonseca $E$. Euthyroid nodular pretibial mucinosis: palliative treatment with carbon dioxide laser. Dermatol Surg. 2009;35:719-21.
MAILING ADDRESS:

Laura de Mattos Milman

Rua Professor Annes Dias, 295

Centro Histórico

90020-090 Porto Alegre, RS.

E-mail:lmmilman@terra.com.br

How to cite this article: Milman LM, Grill AB, Müller GP, De Ville D, Souza PRM. Pretibial mucinosis in an euthyroid patient. An Bras Dermatol. 2016;91(1):100-2. 